\title{
Endonasal Dacryocystorhinostomy, Our Experience
}

\author{
Abdulbaset Naas*, Salem Farhat, Mahmud Darwish and Jamal Ben Naser \\ Consultant Otolaryngologist, Tripoli Medical Centre, Libya
}

*Corresponding author: Abdulbaset Naas, Tripoli Medical center, Libya.

\begin{abstract}
Background: In this study we present our experience of 32 Patients underwent endonasal DCR. They all operated on with one team consisting of Otolaryngologists and an ophthalmologist at Tripoli Medical center.

Objectives: To assess our performance and the success rate. Success was defined as the resolving of symptoms, unobstructed lacrimal system with irrigation and endoscopic visualization of a patent new ostium.

Method: Prospective study of a total 32 patients underwent the endonasal procedure.

Results: A total of 32 microscopic and endoscopic dacryocystorhinostomy performed and reviewed. In 5 cases combined nasal procedures were required. 4 patients $(12,5 \%)$ needed septoplasty and one patient (3\%) needed removal of a Rhinolith). The success rate was $81,3 \%$, there were $15,6 \%$ functional failures and one patient (3\%) needed revision surgery due to recurrent granulation tissue formation at the new ostium region. The follow-up period ranged from 3 months to two years. The procedure achieved $81,3 \%$ success rate.

Conclusion: The described technique of endonasal dacryocystorhinostomy had a success rate comparable to that of external dacryocystorhinostomy, the ease of the operation and the smooth postoperative period with good results, indicates that this surgery is taking more wider place in the management of the nasolacrimal obstruction.
\end{abstract}

Keywords: Dacrocystorhinostomy; DCR; Endoscopic; Endonasal

\section{Introduction}

Toti first describe the external Dacryocystorhinostomy (DCR) in the early 20th century [1], the technique is applicable to patients complaining of tearing and demonstrating obstruction of the lacrimal outflow system. The procedure consists of creating a fistula directly from the lacrimal sac into the nose and bypassing the nasolacrimal duct. Caldwell first described the endonasal approach to the lacrimal sac [2], in 1893, and later in 1911 West introduce it [3], however, its use remained limited due to difficulties in visualizing the endonasal structures during the operation. The introduction of the microscopes and the rigid endoscopes provided the catalyst for endonasal Dacryocystorhinostomy (DCR). In our hospital (Tripoli medical center, Tripoli, Libya), we start this kind of surgery in collaboration with the Ophthalmological department in 2006. We present our experience with 32 patients underwent this operation.

\section{Materials and Methods}

A total of 32 patients with nasolacrimal duct obstruction were treated consecutively by microscopic and endoscopic endonasal DCR. All patients were females (100\%). Patients ages ranged from 22 to 70 years. Patients were usually referred by an ophthalmologist with a history of epiphora and nasolacrimal duct obstruction, all patients has either sicca or post-saccal stenosis. There were 3 cases of Dacryocele, 4 cases needed nasal septal surgery and one patient with rhinolith needed removal of the nasal pathology during the surgery. Pre-operatively, a detailed clinical examination was carried out by an ophthalmologist and an ENT surgeon, including regurgitation testing and lacrimal syringing and probing. Endoscopic evaluation was performed in every case, in order to check for access, deviated nasal septum, turbinate hypertrophy or any other associated pathology. General anesthesia was used in all patients. 


\section{Procedure}

The nose was prepared using cotton strips soaked in 2 percent xylocaine and adrenaline 1:100,000, 10-15 minutes prior to surgery. This ensured adequate decongestion, mucosal anesthesia, easy access and a bloodless field. Two per cent xylocaine with 1:100,000 adrenaline was submucosally injected into the lateral nasal wall, superior and anterior to the attachment of the middle turbinate, and then along the maxillary line. The ocular surface was anaesthetized with two drops of 4 per cent xylocaine. In all patients the endonasal route (West procedure) is used, in 25 (78\%) of Vario ZEISS Microscope is used for magnification, and in 7 (22\%) patients the 0 degree and 30-degree, $4 \mathrm{~mm} \mathrm{STORZ} \mathrm{rigid} \mathrm{endoscopes} \mathrm{were}$ used. First elevation of the flap, an osteotomy was performed with straight Kerrison punch forceps. Sometimes, removal of thick bone from the frontal process of the maxilla was required, for which a drill with 1-2mm diamond burr was used, Lacrimal bone was removed with a Freer`s elevator. At this point the ophthalmologist inserted transcanalicular lacrimal probe through the inferior canaliculus to identify the medial wall of the sac, and/ or external pressure was exerted on the lateral wall just below the medial canthus area, causing the sac to protrude medially in the nasal cavity, and then the sac is opened and part of the medial wall of the sac is removed, syringing done and the silicone tube is inserted and knotted in the nose, (Figure 1), soft nasal pack used for 24 hours, in cases of septoplasty and Rhinolith surgery, nasal splints for 7 days were used to prevent postoperative synechia.

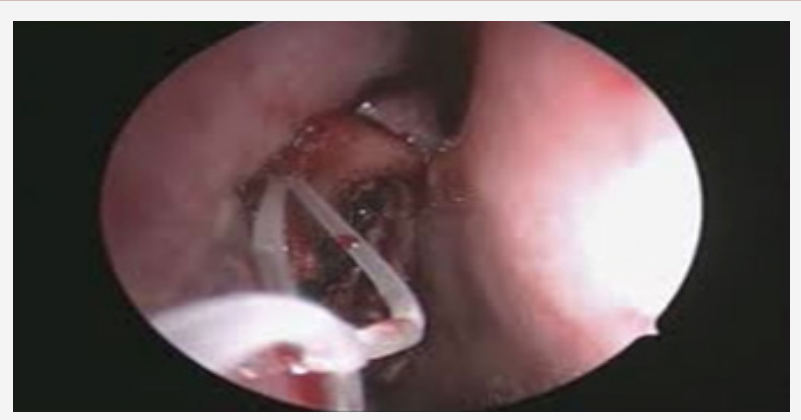

Figure 1: Silicone Tube Knotted in Place (Intraoperative View).

\section{Post-operative care and follow up}

Patients were discharged uneventfully either in the 1st or in the 2 nd postoperative day, and saline nasal drops and antibiotic eye drops were advised to avoid crust formation and in order to ensure continuous flow through the lacrimal system. Patients were advised to avoid nose blowing for one week, to decrease risk of nasal bleeding and orbital emphysema. Patients were followed up after one week, one month and 3 months after surgery. Endoscopic visualization of the nasal cavity was performed in order to remove crusts and granulations (if any) and to check the patency of the newly created ostium using lacrimal irrigation.

The silicone tubes usually removed after 3 months.

\section{Complications}

No major complications were noted.
Minor complications:

1. 2 patients $(6 \%)$ had Intraoperative bleeding and were controlled with bipolar cautery and nasal packing.

2. 2 patients $(6 \%)$ had postoperative lid edema, which was resolved spontaneously.

3. As late complications 3 patients (9\%) developed small nasal synechia, and all needed no therapy as there was no symptoms from it, and one patient (3\%) developed recurrent granulation formation in the region of the new ostium, and this patient needed revision surgery later (Figure 2).

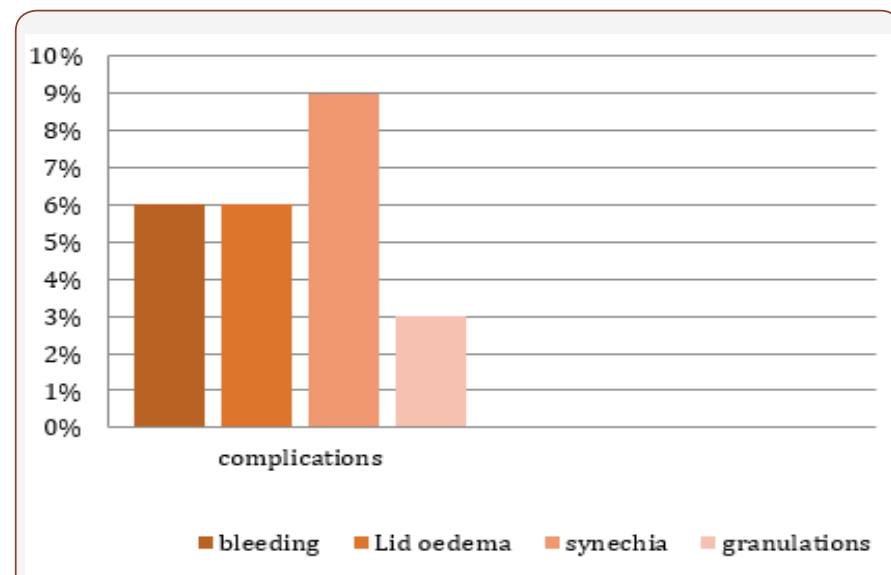

Figure 2: Complications.

\section{Results}

All patients seen 1, 3 and 6 months after surgery for evaluation, and in each visit, they get examined by either ENT specialist or ophthalmologist or both.

In all patients the results were measured

1. Subjectively by questioning the patients.

2. Objectively by using the endoscope and examining the nasolacrimal sac region.

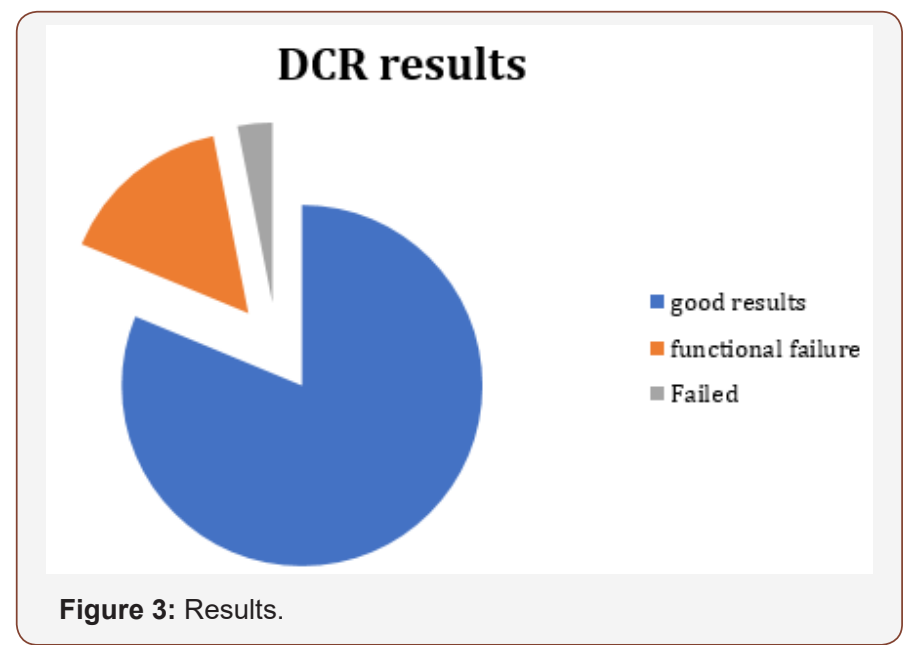

26 patients $(81,3 \%)$ describe the surgery as successful and have no more tearing problems; in those patients the endoscopic examination is normal. 5 patients $(15,6 \%)$ describe the operation 
as unsuccessful and they have still tearing problem, in the nasal endoscopy no specific cause was seen, and they are considered as functional failures. In one failed surgery $(3 \%)$ the cause of failure was a recurrent granulation formation over the tube in the new ostium region; this patient underwent a revision surgery later (Figure 3).

\section{Discussion}

Usually patients complaining from watery eye (Epiphora) seek the ophthalmological advice. This is an unpleasant symptom, which is embarrassing to the patient both socially and functionally. Epiphora is usually caused by obstruction of the upper or lower nasolacrimal system. The endonasal approach is used for the obstructed lower system. Since Toti's original description of DCR in 1904, external approach has been used for relief of the lacrimal obstruction [1]. The success rate of external DCR has improved to the present day with few modifications and may be up to 9095 percent in the hands of a trained oculoplastic surgeon [4]. The advent of microscopic and endoscopic instrumentation for nasal and sinus surgical procedures has prompted renewed interest in the transnasal DCR [5]. The Transnasal approach not only avoids an external incision and scar, but also enhances the surgeon's ability to identify and correct common intranasal causes of DCR failure, including adhesions, an enlarged middle turbinate, nasal septal deviation and paranasal sinus diseases [6]. The main advantage of external DCR is visualization of the anatomy, allowing precise removal of the bone in the lacrimal fossa and exact anastomosis of the nasal mucosa and lacrimal sac wall. Endoscopic surgeons should have a good knowledge of the anatomy of the lacrimal sac and duct within the nose, in order to obtain optimum results comparable to those of external DCR. Endonasal DCR has also been reported to be quicker than the traditional external approach, equivalently successful and preferred by patients [7].

Other alternative is Endonasal laser DCR, which is not the procedure of first preference, due to its high cost, long operation time and less satisfactory results [8] The inferior results achieved with laser DCR may be due to the size of the ostia created; small ostia created by laser DCR have been found to have patency rates of only 64 to 70 percent [4,9]. Endoscopic DCR has many advantages over external DCR (e.g. avoidance of facial scarring, of disruption of lacrimal sac pump action from the orbicularis oculi muscle, and of division of the medial canthal ligament), and it is less traumatic, quicker and cosmetically more convenient, and has a lower complication rate, minimal morbidity and a success rate comparable to traditional external DCR $[4,10]$. The endoscopic approach provides excellent visualization and management of intranasal structures, and it may be associated with improved outcome, considering that intranasal synechiae and improper rhinostomy site placement are common causes of failure of external DCR [6].

The most common causes of failure of endonasal DCR are false localization of the lacrimal sac, granulation tissue formation, retained bony specula, inadequate removal of the medial sac wall, and synechiae between the lateral wall and the middle turbinate [11]. In our view, appropriate post-operative care is essential to prevent endonasal synechiae and subsequent recurrences. Kong et al., reported granulation at the internal nasal opening in approximately 50 per cent of patients at eight weeks post-operatively $[10,12]$. Endoscopic transnasal DCR is a valid alternative to the traditional extranasal procedure. However, this technique does require a certain expertise in endoscopic surgery. Adequate removal of bone; creating a flap of lacrimal sac mucosa and approximating it to the nasal mucosa; and regular post-operative endoscopic follow up to remove crusts, synechiae and granulations. Creation of a wide surgical window helps ensure a success rate comparable to that of the external procedure.

In most cases, sophisticated equipment is not required, and the procedure can be performed with just a few, routinely used endoscopic sinus surgery instruments, in our study, we have found females predominance, which is higher than in the other studies $[5,7,9,11]$. Our success rate in this study is $81.3 \%$, which can be compared with other studies. Literature review shows that the success rate of endoscopic dacryocystorhinostomy varies between 80 to $95 \%[13,14]$. We have in our series of 32 patient one clear functional and anatomical failure (3\%), due to repeated Granulation tissue formation, Meticulous cleaning of the granulation tissue at regular follow-up visits and regular use of saline sprays by the patients are essential to prevent Granulation tissue and synechia formation, also use of the silicone tubes for 3 months should prevent early closure or synechia at the nasolacrimal sac region.

\section{Conclusion}

Endonasal DCR surgery offers a very attractive alternative to the well-established technique of external DCR surgery for the treatment of primary acquired nasolacrimal duct obstruction with comparable success rates, shorter surgical time and higher patient satisfaction. Endonasal surgery may have a benefit of preserving the lacrimal pump system and leaving no scar. Patient preference and availability of each service should direct the management; hence endonasal DCR surgery should be considered for primary treatment of nasolacrimal duct obstruction.

\section{Acknowledgement}

None.

\section{Conflict of Interest}

No conflict of interest.

\section{References}

1. Toti A (1904) A new conservative method of dacryocystorhinostomy for chronic suppuration of lacrimal sac [in Italian]. Clin Mod (Firenza) 10: 385-387.

2. Caldwell GW (1893) Two new operations for obstruction of the nasal duct, with preservation of the canaliculi, and with an incidental description of a new lacrimal probe. Am J Ophthalmol 10: 189-193.

3. West JM (1910) A window resection of the nasal duct in cases of stenosis. Trans Am Ophthalmol Soc 12: 654-658.

4. Hartikainen J, Antila J, Varpula M, Puukka P, Seppa H, et al. (1998) Prospective randomised comparison of endonasal endoscopic 
dacryocystorhinostomy and external dacryocystorhinostomy. Laryngoscope 108: 1861-1866.

5. Metson R (1990) Endoscopic approach for revision dacryocystorhinostomy. Laryngoscope 100: 1344-1347.

6. Metson R (2001) Dacryocystorhinostomy. In: Kennedy DW, Bolger WE, Zinreich SJ (Eds.), Disease of the Sinuses, Diagnosis and Management. Hamilton: BC Decker pp: 317-324.

7. Dolman PJ (2003) Comparison of external dacryocystorhinostomy with nonlaserendonasal dacryocystorhinostomy. Ophthalmology 110: 78-84.

8. Onerci M (2002) Dacryocystorhinostomy. Diagnosis and treatment of nasolacrimal canal obstructions. Rhinology 40: 49-65.

9. Hehar SS, Jones NS, Sadiq A, Downes RN (1997) Endoscopic holmium: YAG laser dacryocystorhinostomy safe and effective as a day case procedure. J Laryngol Obol 111: 1056-1059.
10. Cokkeser Y, Evereklioglu C, Er H (2000) Comparative external versus endoscopic dacryocystorhinostomy: result in 115 patients (130 eyes). Otolaryngol Head Neck Surg 123: 488-491.

11. Onerci M, Orhan M, Ogretmenoglu O, Irkec M (2000) Long- term results and reasons for failure of intranasal endoscopic dacryocystorhinostomy. Acta Otolaryngol 120: 319-322.

12. Kong YT, Kim TI, Kong BW (1994) A report of 131 cases of endoscopic laser lacrimal surgery. Ophthalmology 101: 1793-1800.

13. Yung MN, Hardman Lea S (2002) Analysis of the results of surgical endoscopic dacryocystorhinostomy: Effect of the level of obstruction. $\mathrm{Br}$ J Ophthalmol 86(7): 792-794.

14. Wormald PJ (2002) Powered endoscopic dacryocystorhinostomy. Laryngoscope 112(1): 69-72. 